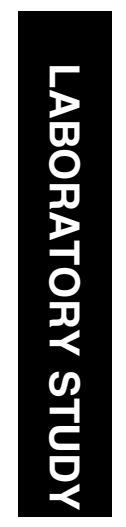

${ }^{1}$ Section of Clinical Epidemiology, Institute of Epidemiology and Social Medicine, University of Muenster, Muenster, Germany

${ }^{2}$ Department of Genetic Epidemiology of Vascular Disorders, Leibniz Institute of Arteriosclerosis Research, University of Muenster, Muenster, Germany

${ }^{3}$ Institute of Human Genetics, University of Regensburg, Regensburg, Germany

${ }^{4}$ Department of Ophthalmology, St Franziskus Hospital, Muenster, Germany

Correspondence: $\mathrm{H}-\mathrm{W}$ Hense, Section of Clinical Epidemiology, Institute of Epidemiology and Social Medicine, 3, Domagk Street, 48129 Muenster, Germany

Tel: +49251835 5399;

Fax: + 492518355300

E-mail: hense@

uni-muenster.de

Received: 5 September 2008

Accepted in revised form: 16 December 2008

Published online:

23 January 2009

This study was supported in part by grants from the Deutsche

Forschungsgemeinschaft $\mathrm{He}$ 2293/5-1, 5-2, 5-3; We 1259/14-3, the intramural IMF fund of the University of Muenster, and the Pro Retina Foundation

\section{Variations in five genes and the severity of age- related macular degeneration: results from the Muenster aging and retina study}

\author{
A Farwick ${ }^{1,2}$, B Dasch ${ }^{1}$, BHF Weber ${ }^{3}$, \\ D Pauleikhoff ${ }^{4}, \mathrm{M} \mathrm{Stoll}^{2}$ and $\mathrm{H}-\mathrm{W}$ Hense
}

\begin{abstract}
Aims Little is known about the role of genetic variants in the early stages of age-related macular degeneration (AMD). We aimed to investigate how genetic variations within five well-defined genes relate to AMD severity.

Methods We analysed SNPs in the genes for complement factor $\mathrm{H}(\mathrm{CFH})$, age-related maculopathy susceptibility (ARMS2), HtrA

Conclusion Our findings indicate that the CFH gene is involved in the onset of AMD, whereas both, the $C F H$ and $A R M S 2$ genes, and more weakly, the HtrA1 gene, appear to account for the advancement of AMD. The results for SNPs in the $C 2$ and $C F B$ genes were inconclusive. Genetic factors dominated in their impact over age and smoking history. Eye (2009) 23, 2238-2244; doi:10.1038/eye.2008.426; published online 23 January 2009
\end{abstract} serine peptidase 1 (HtrA1), complement factor $B$ (CFB), and complement component 2 (C2) in 183 controls and 730 patients with increasing severity of AMD from the Muenster aging and retina study (MARS). Severity scoring was based on the Rotterdam classification of fundus photographs.

Results Compared with controls, patients with very early AMD showed a significantly increased minor allele frequency (MAF) only for $C F H$-rs1061170. With increasing severity of AMD, SNPs in CFH-rs1061170, as well as ARMS2-rs10490924, became consistently more common $(P<0.001)$. Likewise, HtrA1rs11200638 was less clearly associated with AMD severity, whereas C2-rs9332739 and CFB-rs641153 showed no relation. Multifactorial models confirmed $\mathrm{CFH}$ and ARMS2 as major determinants of AMD severity, whereas addition of $\mathrm{HtrA1}, \mathrm{C} 2$ and $C F B$ did not improve model prediction. In the models, age did not contribute to very early but to all more severe AMD stages, whereas smoking history had a significant impact only for late AMD.
Keywords: age-related macular degeneration; severity; CFH; ARMS2; HtrA1; C2; CFB

Introduction

Age-related macular degeneration (AMD) is a chronic disease of the retina and the leading cause of visual impairment and legal blindness among older people in industrialised countries. ${ }^{1,2}$ The early stages of AMD are characterised by intermediate or large soft drusen and hyper- or hypopigmentation of the retinal pigment epithelium with usually only minor or no clinical symptoms. ${ }^{3,4}$ Extension, type, and bilateral concurrence of morphologic alterations of the retina are closely related to the risk of progression to advanced AMD, geographic atrophy (GA), and choroidal neovascularisation $(\mathrm{CNV}){ }^{5}$ which commonly cause vision impairment and blindness.

Numerous studies have indicated that AMD is a complex, multifactorial disease with a prominent genetic component despite a disease onset late in life. ${ }^{4,6}$ Common single nucleotide 
polymorphisms (SNPs) in the complement factor $H$ (CFH) gene on chromosome $1,^{7-10}$ and in two loci on chromosome 10q26, that is, ARMS2 (formerly labelled as LOC387715) ${ }^{11-15}$ and the HtrA serinepeptidase 1 (HtrA1) gene $^{16-19}$ showed strong associations with the risk of advanced AMD. These results have been extensively replicated in studies conducted in various populations and settings (for an overview, see Jakobsdottir et $a l^{20}$ ). Additional studies investigated the complement pathways in more detail. Their findings indicated that genes coding for other elements of the complement system, such as component $2(C 2)$ and complement factor $B$ $(C F B)$ on chromosome $6 \mathrm{p}$, might be involved in a protective manner. ${ }^{20-23}$

As emphasised by Jakobsdottir et $a l^{20}$ many genetic association studies have compared cases of advanced AMD with eye-healthy controls to enhance phenotypic contrast. Much less is known about the role of genetic variants for AMD onset and the course of the early forms of AMD. Some studies have included groups of individuals with conditions labelled as early $\mathrm{AMD}^{23,24}$ or low-risk $\mathrm{AMD}^{12}$ whereas others investigated in more detail how genetic variants relate to specific morphologic features of early AMD. ${ }^{12,24-29}$ These studies reported that the allelic risk variants of the $\mathrm{CFH}$ gene were more frequent among early AMD cases compared with controls but that the risk was much stronger among patients with more advanced disease. ${ }^{12,24-26,29}$ Similarly, Rivera et al $^{12}$ showed for the ARMS2-rs10490924 variant, a slightly higher frequency in patients with early AMD, whereas substantially increased frequencies were observed in patients with late AMD. Recently, it was hypothesised that genetic variations in the $C F H$ locus contributed to global and diffuse retinal disease, including early maculopathy and extramacular parts of the retina, while the ARMS2 variant and HtrA1rs11200638 appeared mainly related to the angiogenic pathway and specifically to neovascularisation. ${ }^{16,17,28,30,31}$ Moreover, some new studies suggested that SNPs in the $C 2-C F B$ locus may also effect the development of early signs of AMD, such as drusen formation due to a modified activity of the complement cascade. ${ }^{20,21,23}$

We present here analyses on a sample of 183 controls and 730 individuals who were classified into four groups according to rising severity of AMD, and we investigated how the occurrence of allelic variants in the $C F H$, ARMS2, HtrA1, C2, and CFB genes differed across these stages of severity.

\section{Materials and methods}

\section{Study population}

The Muenster aging and retina study (MARS) is a longitudinal study designed to identify medical, environmental, and genetic factors with implications for the progression of AMD. From June 2001 to October 2003, a cohort of 1060 individuals from the city of Muenster and the surrounding counties was assembled. ${ }^{32-34}$ All participants were of Caucasian origin. A cross-sectional analysis of this baseline examination is the basis of the present report.

Study subjects were eligible as AMD patients for inclusion to MARS when they had morphologic evidence of early AMD in at least one eye and if age at diagnosis was between 60 and 80 years. Individuals with severe cataract, a diagnosis of narrow-angle glaucoma or with severe myopia were not eligible. Control subjects were recruited on an opportunistic basis from volunteers, spouses, and unrelated companions of the AMD cases if they agreed to a mydriatic ophthalmic examination, which showed no signs of AMD after grading their fundus photographs in a masked way.

The recruitment and research protocols were reviewed and approved by the Institutional Review Board of the University of Muenster, and written informed consent was obtained from all study participants.

\section{Examination procedures and fundus photography}

Examination procedures and fundus photography have been previously described in detail. ${ }^{32}$ All digital images were evaluated on a 22-inch computer screen by specially trained technicians. The presence and severity of retinal lesions were graded according to the protocol of the International age-related maculopathy (ARM) Epidemiological Study Group. ${ }^{3}$

We graded each eye of a study individual independently, and we included individuals in this report only when the grading in both eyes was possible. We employed the fundus grading system of the International ARM Epidemiological Study Group ${ }^{3}$ for AMD. In accordance with the Rotterdam study classification, ${ }^{2}$ the range of fundus signs was stratified into a five severity stages: stage 0 , no sign of ARM or hard drusen ( $<63 \mu \mathrm{m})$ only; stage 1 , soft distinct drusen $(\geqslant 63 \mu \mathrm{m})$ only, or pigment epithelium changes only, no soft drusen $(\geqslant 63 \mu \mathrm{m})$; stage 2 , soft indistinct drusen $(\geqslant 125 \mu \mathrm{m})$ or reticular drusen only, or soft distinct drusen $(\geqslant 63 \mu \mathrm{m})$ with pigment epithelium changes; stage 3 , soft indistinct drusen $(\geqslant 125 \mu \mathrm{m})$ or reticular drusen with pigment epithelium changes; and stage 4, atrophic or neovascular AMD. Subjects were classified according to the stage in the eye with the worse finding.

\section{DNA extraction, SNP selection, and genotyping}

We extracted DNA from peripheral blood by standard methods. Variants within the CFH (rs1061170, rs2274700, and rs3766404), ARMS2 (rs10490924 and rs10490923), 
HtrA1 (rs11200638), CFB (rs12614, rs547154, rs641153, rs1048709, and rs2072633), and C2 (rs4151659, rs4151667, and rs9332739) genes were genotyped. For genotyping, we used 2 ng genomic DNA and Taqman SNP Genotyping Assays (Applied Biosystems, Foster City, CA, USA) following the manufacturers' instructions.

The polymerase chain reaction (PCR) was specifically employed to characterise the three SNPs rs11200638 (HtrA1), rs641153 (CFB), and rs12614 (CFB). We used the oligonucleotide primers $5^{\prime}$ CACCCTCGCCAGTTACGA $3^{\prime}$ (sense) and 5' GGGGAAAGTTCCTGCAAA $3^{\prime}$ (antisense) for the variant in the $H \operatorname{tr} A 1$ gene $5^{\prime}$ CCTTCCTGACAGTCTTTTGGTC $3^{\prime}$ (sense) and 5' CTTCTCTCCTGCCTTCCAAC $3^{\prime}$ (antisense) for the two SNPs in the CFB gene. PCR was carried out with the TaqPCR Core Kit from Qiagen following the manufacturers' instructions. DNA sequencing was performed using the Big Dye Terminator cycle sequencing method on an automated DNA sequencer (ABI3730, Applied Biosystems). Sequence tracks were analysed by software sequencing analysis 5.2 (Applied Biosystems). Genotyping efficacy was $>97 \%$. Estimation of the Hardy-Weinberg equilibrium (HWE) and fine mapping of the ARMS2/HtrA1 region was performed with Haploview. ${ }^{35}$

We investigated the frequencies of 14 SNPs in five genes. We excluded three SNPs (rs3766404, rs10490923, and rs547154) from further analyses as their genotyping call rates were $<90 \%$. For the sake of conciseness and clarity, we further present results only for one SNP from each gene. Therefore, CFH-rs2274700, C2-rs4151667, and C2-rs4151659, as well as CFB-rs12614, CFB-rs1048709, and $C F B$-rs2072633 were excluded because the association for each of these variants with AMD severity stages was less strong than for the SNPs retained in this report.

\section{Statistical analyses}

Of the 1060 participants of the MARS baseline examination, digital fundus images were not obtained for the following reasons: refusal (two patients), technical problems of the retina camera (5 right and 15 left eyes), severe opacities of the lens or cornea in one eye (six right and five left eyes), and insufficient dilatation (two right and five left eyes). Because of other adverse eye conditions (eg, gliosis, diabetic retinopathy, and so on), the AMD grade could not be assessed in 44 right and 40 left eyes. Furthermore, no DNA was provided by 51 participants and genotyping was not successful in another 11 subjects. Thus, genotyping and gradable digital fundus photographs in both eyes were available for 913 subjects (86\% of all study participants).

We defined genotypes as categorical variables with three levels. We used logistic regression models to assess the association of single SNPs with AMD severity level under the assumption of an additive model on a log scale as also used by others. ${ }^{12,14,31,36}$ In a first step, odds ratios (ORs) were computed for one copy of the risk allele separately for each SNP. The statistical power of each severity group was calculated for every SNP using the freeware online tool 'Genetic Power Calculator'. ${ }^{37} \mathrm{We}$ assessed the contributions of variants in different genes at each severity stage by fitting logistic regression models that included the CFH variant (rs1061170), and, in consecutive order, additionally ARMS2-rs10490924, HtrA1-rs11200638, C2-rs9332739, and CFB-rs641153. Finally, age, gender, and smoking status were included to identify the independent impact of these non-genetic variables on AMD severity. Model performance was evaluated using the area under the ROC curve (or $c$-statistics) to assess the discriminatory power of the model. ${ }^{38}$ To account for the number of statistical comparisons performed for each SNP, we adjusted the statistical significance level to the number of AMD stages $(\alpha / n$, ie, $0.05 / 4=0.0125)$, and report exclusively $99 \%$ confidence intervals. Likewise, statistical significance of a result was accepted if the error level was less than $1 \%$. All analyses were performed using STATA 9.0 and SAS 9.1.

\section{Results}

\section{Baseline description of the study sample}

The control group in our study sample consisted of 183 individuals who had no signs of AMD. The cases with advancing severity of AMD among the MARS participants were similar to those with different levels of maculopathy, although there were 280 individuals with late AMD in at least one eye (Table 1). Patients with more severe AMD were older and they had more often smoked during their lifetime. The proportion of women in each stage ranged from 54 and $70 \%$.

\section{AMD severity stages and genetic variants}

Figure 1 shows the minor allele frequencies (MAFs) of the five SNPs in groups of AMD severity. All variants

Table 1 Baseline characteristics of the study participants in AMD severity groups

\begin{tabular}{lcccc}
\hline $\begin{array}{l}\text { AMD } \\
\text { severity }\end{array}$ & $\mathrm{N}$ & $\begin{array}{c}\text { Ever smoked } \\
(\%)\end{array}$ & $\begin{array}{c}\text { Women } \\
(\%)\end{array}$ & $\begin{array}{c}\text { Mean age } \\
\text { (years) }\end{array}$ \\
\hline 0 & 183 & 30.1 & 63.3 & 68.5 \\
1 & 194 & 40.7 & 55.7 & 70.2 \\
2 & 135 & 35.9 & 63.0 & 71.5 \\
3 & 121 & 36.7 & 70.3 & 71.6 \\
4 & 280 & 50.2 & 53.6 & 72.3 \\
Total & 913 & 40.2 & 59.6 & 70.9 \\
\hline
\end{tabular}




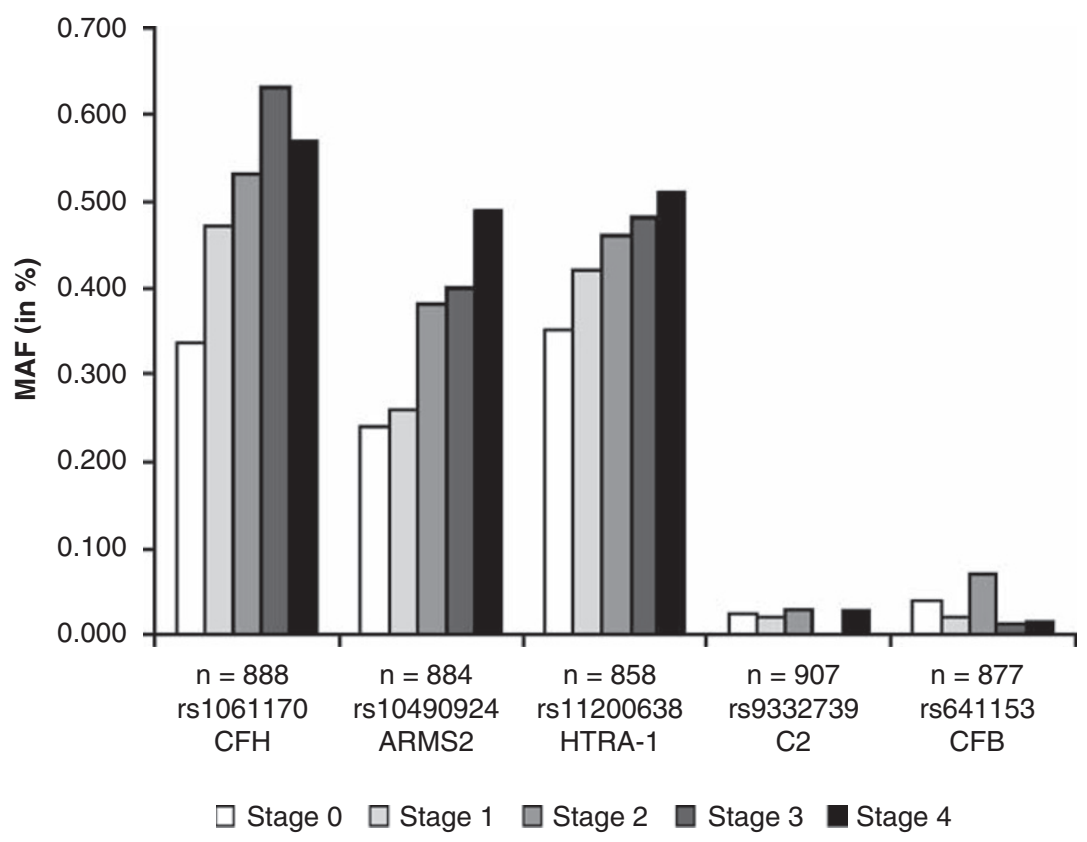

Figure 1 Minor allele frequencies (MAFs, in \%) for five SNPs in AMD severity stage.

Table 2 Association of allelic variants in five genes with AMD severity, presented as odds ratios and respective $99 \%$ confidence intervals

\begin{tabular}{|c|c|c|c|c|c|c|c|c|}
\hline \multirow[t]{2}{*}{ Gene } & \multirow[t]{2}{*}{ ref. SNP ID } & \multirow[t]{2}{*}{ Amino-acid change } & \multirow[t]{2}{*}{ Total $\mathrm{N}$} & \multicolumn{5}{|c|}{ AMD severity (OR $(99 \%$ CI)) } \\
\hline & & & & 0 & 1 & 2 & 3 & 4 \\
\hline $\mathrm{CFH}$ & rs1061170 & $\mathrm{Y} 402 \mathrm{H}$ & 888 & Ref. & $1.9(1.2-2.9)$ & $2.3(1.5-3.6)$ & $3.5(2.1-5.7)$ & $2.5(1.7-3.7)$ \\
\hline ARMS2 & rs10490924 & A69S & 884 & Ref. & $1.1(0.7-1.7)$ & $1.8(1.2-2.9)$ & $2.2(1.3-3.6)$ & $2.6(1.8-3.7)$ \\
\hline HTRA1 & rs11200638 & Promoter & 858 & Ref. & $1.3(0.9-1.9])$ & $1.6(1.04-2.5)$ & $1.7(1.1-2.6)$ & $1.9(1.3-2.7)$ \\
\hline $\mathrm{C} 2$ & rs9332739 & E318D & 907 & Ref. & $0.9(.3-3.6)$ & $1.4(0.5-4.1)$ & $1.1(0.3-3.7)$ & $0.9(0.2-3.5)$ \\
\hline CFB & rs641153 & R32Q & 877 & Ref. & $0.6(0.2-1.9)$ & $0.3(0.03-1.6)$ & $0.4(0.07-1.9)$ & $0.4(0.1-1.4)$ \\
\hline
\end{tabular}

Odds ratio estimates from logistic regression models are presented for one copy of the risk allele; estimates for homozygotes can be obtained by taking the square of these odds ratios (under the assumption of a genetic model with additive effects on a log scale).

Bold values $=$ statistically significant with error probability $\alpha<1 \%$.

were in HWE in the controls. Although SNPs in the $C F H$ $(\mathrm{MAF}=0.336)$, ARMS2 (0.240), and HtrA1 (0.351) genes were common among controls, SNPs in the $C 2(0.024)$ und $C F B(0.040)$ genes were quite rare (Figure 1 and Supplementary Table). The MAF rose consistently with AMD stage for CFH, ARMS2, and HtrA1 (test for trend, $P<0.001)$ but no clear relation emerged for $C 2$ and $C F B$ (Supplementary Table).

These associations were generally confirmed when fitting separate logistic regression models (Table 2). We found that in stage 1, CFH-rs1061170 was the only SNP with a significantly elevated OR, and that the magnitude of the ORs increased in a graded manner up to the higher severity levels. By comparison, the OR for ARMS2-rs10490924 and for HtrA1-rs11200638 was not significantly raised in stage ' 1 ', but from stage ' 2 ' upwards, a graded risk increase was observed. The impact of HtrA1-rs11200638 seemed less pronounced than for ARMS2-rs10490924 (maximum OR: 1.9 vs 2.6).

To further clarify the association between ARMS2 and HtrA1, we did a fine mapping of this region, that revealed only a low linkage disequilibrium between ARMS2-rs10490924 and HtrA1-rs11200638 $\left(D^{\prime}=0.44\right)$.

Moreover, the OR for C2-rs9332739 oscillated around unity across AMD severity levels and showed no statistical significance. Although a consistent tendency towards a protective effect was found for CFB-rs641153, the individual ORs (ranging from 0.3 to 0.6 ) did not reach statistical significance.

\section{Multifactorial models}

The logistic regression models that included only one SNP generally contributed rather moderately to the 
explanation of the AMD severity level. Thus, for CFH-rs1061170, the values of the $c$-statistics (area under the ROC curve) were $c=0.62$ for AMD stage $1, c=0.61$ (stage 2), $c=0.64$ (stage 3 ), and $c=0.67$ (stage 4). Addition of ARMS2-rs10490924 improved the discriminatory performance of the model significantly $(P<0.001)$ : $c$-statistics were now $0.60,0.67,0.75$, and 0.74 , respectively. Further addition of HtrA1-rs11200638 barely improved these results (c-statistics were 0.62, 0.68, 0.76, and 0.75 , respectively). Likewise, addition of SNPs from the $C 2$ and $C F B$ gene contributed even less to the $c$-statistics of the models. Including age, sex, and ever smoking in a model containing CFH-rs1061170, ARMS2-rs10490924, and HtrA1-rs11200638, improved the model performance in a marked and significant way for all stages ( $c=0.66,0.74,0.80$, and 0.81 , respectively). This was mostly due to significant impacts of age in these models, whereas the effect of smoking history was of only significance in stage 4 patients. Gender had no significant influence at any AMD severity level.

\section{Discussion}

In this cross-sectional analysis, we took a focused view on how genotypes relate to the different stages of AMD severity. We found that only the SNP CFH-rs1061170 was related to the earliest AMD stages. These results are in agreement with earlier reports, ${ }^{24-26,39}$ and indicate that the $C F H$ gene seems to play a key role already in the onset of AMD. SNPs from both, the CFH and the ARMS2 gene, were more common among patients with more severe AMD. The increments with every severity stage were steady and strong; ORs for the presence of a single allele copy increased substantially in the late stages of AMD. We view the graded rise as evidence of the relative importance of the two SNPs in the progression of AMD.

HtrA1-rs11200638 showed significant effects, but of lower strength, and its contribution in the multifactorial models was insignificant. This result is not supporting an independent role of this variant as claimed by other reports. ${ }^{31,36}$ Fine mapping of the ARMS2/HtrA1 region revealed a low linkage disequilibrium between ARMS2-rs10490924 and HtrA1-rs11200638 $\left(D^{\prime}=0.44\right)$ supporting the hypothesis that ARMS-rs10490924 represents the major susceptibility polymorphism of this region and that HtrA1-rs11200638 is only indirectly associated with AMD. ${ }^{14,15}$

Our analysis of the C2-CFB-locus was inconclusive. The risk variants C2-rs9332739 and CFB-rs641153 were rare in the MARS study sample and appeared unrelated to AMD severity. Nevertheless, our results do not rule out the protective role-especially of $C F B$ rs641153 - that was claimed by others, ${ }^{20-23}$ because the statistical power of our study was too low to provide conclusive confirmation or refutation of these results. The inclusion of $C 2 / C F B$ in multifactorial analyses did also not significantly improve the prediction contrary to the finding of Jakobsdottir et al. ${ }^{20}$ We note, however, that these authors used a different SNP in the CFB gene (rs4151667) while confirming our result of no association of C2-rs9332739 with AMD.

Our analytic approach compares the proportions of individuals who carry at least one copy of the respective SNP in each severity level. We note that this proportion depends, aside from sampling variation, also on the relative impact of other causative factors. We assumed that, under a model of component causes, the proportion of carriers of the risk allele in each severity stage is an indicator of the relative importance of this SNP within a sufficient cause. ${ }^{40}$

Presently, it is not clearly understood how genetic factors influence the onset and advancement of AMD, ${ }^{6}$ and it has to be noted that the variants in the five genes studied here exert various functions and are involved in different biological processes and/or pathways. $C F H$, for example, is the major inhibitor of the alternative complement cascade, ${ }^{41,42}$ and has previously been associated with drusen formation. ${ }^{25,26}$ The function of the ARMS2 gene was poorly understood ${ }^{6,12}$ until recently when Fritsche et $a l^{14}$ could show that the expression of ARMS2 is present in mitochondria of cells among others of the human retina. In line with recent findings of Kanda et $a{ }^{15}$ they propose that ARMS2 has a key role in AMD, possibly through mitochondrial-related pathways. ${ }^{14}$ Presently, the role of HtrA1, located very close to ARMS2 on 10q26 and coding for a well-characterised heat-shock protease, is less clear. Evidence for a significant role of HtrA1 in AMD was proposed by Yang et al ${ }^{17}$ who showed a positive HtrA1 staining in drusen and found an enhanced HtrA1 expression in the RPE of AMD patients carrying the rs11200638 variant. C2 and CFB are paralogous genes on chromosome $6 \mathrm{p} 21$ that aid the initiation of the complement cascade. The key role of $C 2$ and $C F B$ in AMD pathobiology seems to be the regulation of the complement activation, and hence the influence in inflammatory processes. ${ }^{20,21,23}$ Finally, the contribution of non-genetic factors in our study was modest and confined to age and, less markedly, to smoking history. We noted a significant influence only in the later stages of AMD.

There are limitations to our study. First, MARS is not a population-based sampling, and used as a clinical convenience sampling. However, all tested SNPs were in HWE, especially in our control group, indicating that our reference group does not appear to be grossly biased by selection. We compared the MAF and the proportions of genotypes in our control group with reports from the literature representing European populations of 
Caucasian descent. We found that the MAF for CFH-rs1061170, ARMS2-rs1049024, and C2-rs9332739 were in very good agreement with reports from population-based samples. ${ }^{12,20,36,43}$ Hence, the reported ORs can be assumed to be unbiased. On the other hand, we found significantly higher MAF for HtrA1-rs11200638 and clearly lower MAF for CFB-rs641153 compared with other populations ${ }^{21,23,31,36}$ supposedly due to the different genotyping methods used. We employed the resequencing method, which is regarded as the goldstandard of genotyping methods. Therefore, we suppose that these MAF in our control group are valid and that it is unlikely that they are based on a sampling error.

Second, controls in our study may still develop AMD later in life. Although this is true, we do not conceive that this invalidates our analytic approach; stages of severity in an age-related disease imply, by definition, that the mean years of age differ by stage. We suggest that causal contributions may be inferred when the exposure is a biologically invariable factor (eg, genetic makeup), and selective survival or participation bias can be plausibly excluded. We are not aware that the genetic traits evaluated here confer any survival disadvantage, nor can we conceive of any selective participation conditional on the genotypes.

Third, examination methods, such as angiography, are better for the accurate diagnosis of late stage AMD, especially the differential diagnosis of CNV, than fundus photos. However, these more laborious examination methods are difficult to obtain in larger scale epidemiological studies, and therefore, most study results concerning the association of polymorphisms and AMD are, like MARS, restricted to fundus photos. Fourth, statistical power analysis revealed a $90 \%$ power to detect an association at $\alpha=0.05$ through the transmission disequilibrium test analysis for the risk variants of CFH-rs1061170, ARMS2-rs10490924, and HtrA1-rs11200638 at every severity stage. On the other hand, the statistical power was insufficient for the minor alleles in the $C 2$ and $C F B$ genes to detect significant associations, as also denoted above. We do not believe, however, that these low MAF are biased by sampling errors but that they correctly reflect the situation in this population from the Northwest of Germany.

\section{Conclusion}

Our findings support the notion that the $\mathrm{CFH}$ gene is involved in the onset of AMD, although both, the $C F H$ and ARMS2 genes, and more weakly the HtrA1 gene, account for the advancement of AMD. The results of SNPs in the $C 2$ and $C F B$ genes were inconclusive. Genetic factors dominated in their impact on AMD severity over non-genetic factors, that is, age and smoking history. Clearly, prospective studies are needed to further clarify the impact of genetic factors on AMD progression. We hope that the prospective analyses of the MARS cohort, which are presently under way, will be able to contribute to this clarification.

\section{References}

1 de Jong PT. Age-related macular degeneration. N Engl J Med 2006; 355: 1474-1485.

2 van Leeuwen R, Chakravarthy U, Vingerling JR, Brussee C, Hooghart AJ, Mulder PG et al. Grading of age-related maculopathy for epidemiological studies: is digital imaging as good as 35-mm film? Ophthalmology 2003; 110: 1540-1544.

3 Bird AC, Bressler NM, Bressler SB, Chisholm IH, Coscas G, Davis $\mathrm{MD}$ et al. An international classification and grading system for age- related maculopathy and age-related macular degeneration. The International ARM Epidemiological Study Group. Surv Ophthalmol 1995; 39: 367-374.

4 de Jong PT, Bergen AA, Klaver CC, van Duijn CM, Assink JM. Age-related maculopathy: its genetic basis. Eye 2001; 15: 396-400.

5 Ferris FL, Davis MD, Clemons TE, Lee LY, Chew EY, Lindblad AS et al. A simplified severity scale for age-related macular degeneration: AREDS report no. 18. Arch Ophthalmol 2005; 123: 1570-1574.

6 Scholl HP, Fleckenstein M, Issa PC, Keilhauer C, Holz FG, Weber $\mathrm{BH}$. An update on the genetics of age-related macular degeneration. Mol Vis 2007; 13: 196-205.

7 Edwards AO, Ritter IR, Abel KJ, Manning A, Panhuysen C, Farrer LA. Complement factor $\mathrm{H}$ polymorphism and agerelated macular degeneration. Science 2005; 308: 421-424.

8 Hageman GS, Anderson DH, Johnson LV, Hancox LS, Taiber AJ, Hardisty LI et al. A common haplotype in the complement regulatory gene factor $\mathrm{H}$ (HF1/CFH) predisposes individuals to age-related macular degeneration. Proc Natl Acad Sci USA 2005; 102: 7227-7232.

9 Haines JL, Hauser MA, Schmidt S, Scott WK, Olson LM, Gallins $\mathrm{P}$ et al. Complement factor $\mathrm{H}$ variant increases the risk of age-related macular degeneration. Science 2005; 308: 419-421.

10 Klein RJ, Zeiss C, Chew EY, Tsai JY, Sackler RS, Haynes C et al. Complement factor $\mathrm{H}$ polymorphism in age-related macular degeneration. Science 2005; 308: 385-389.

11 Jakobsdottir J, Conley YP, Weeks DE, Mah TS, Ferrell RE, Gorin MB. Susceptibility genes for age-related maculopathy on chromosome 10q26. Am J Hum Genet 2005; 77: 389-407.

12 Rivera A, Fisher SA, Fritsche LG, Keilhauer CN, Lichtner P, Meitinger Tet al. Hypothetical LOC387715 is a second major susceptibility gene for age-related macular degeneration, contributing independently of complement factor $\mathrm{H}$ to disease risk. Hum Mol Genet 2005; 14: 3227-3236.

13 Schmidt S, Hauser MA, Scott WK, Postel EA, Agarwal A, Gallins $\mathrm{P}$ et al. Cigarette smoking strongly modifies the association of LOC387715 and age-related macular degeneration. Am J Hum Genet 2006; 78: 852-864.

14 Fritsche LG, Loenhardt T, Janssen A, Fisher SA, Rivera A, Keilhauer $\mathrm{CN}$ et al. Age-related macular degeneration is associated with an unstable ARMS2 (LOC387715) mRNA. Nat Genet 2008; 40: 892-896.

15 Kanda A, Chen W, Othman M, Branham KE, Brooks M, Khanna $\mathrm{R}$ et al. A variant of mitochondrial protein 
LOC387715/ARMS2, not HTRA1, is strongly associated with age-related macular degeneration. Proc Natl Acad Sci USA 2007; 104: 16227-16232.

16 Dewan A, Liu M, Hartman S, Zhang SS, Liu DT, Zhao C et al. HTRA1 promoter polymorphism in wet age-related macular degeneration. Science 2006; 314: 989-992.

17 Yang Z, Camp NJ, Sun H, Tong Z, Gibbs D, Cameron DJ et al. A variant of the HTRA1 gene increases susceptibility to age-related macular degeneration. Science 2006; 314: 992-993.

18 Gibbs D, Yang Z, Constantine R, Ma X, Camp NJ, Yang X et al. Further mapping of 10q26 supports strong association of HTRA1 polymorphisms with age-related macular degeneration. Vision Res 2008; 48: 685-689.

19 Yoshida T, Dewan A, Zhang H, Sakamoto R, Okamoto H, Minami M et al. HTRA1 promoter polymorphism predisposes Japanese to age-related macular degeneration. Mol Vis 2007; 13: 545-548.

20 Jakobsdottir J, Conley YP, Weeks DE, Ferrell RE, Gorin MB. $\mathrm{C} 2$ and $\mathrm{CFB}$ genes in age-related maculopathy and joint action with CFH and LOC387715 genes. PLoS ONE 2008; 3: e2199.

21 Spencer KL, Hauser MA, Olson LM, Olson LM, Schmidt S, Scott WK et al. Protective effect of complement factor B and complement component 2 variants in age-related macular degeneration. Hum Mol Genet 2007; 16: 1986-1992.

22 Maller J, George S, Purcell S, Fagerness J, Altshuler D, Daly MJ et al. Common variation in three genes, including a noncoding variant in $\mathrm{CFH}$, strongly influences risk of age-related macular degeneration. Nat Genet 2006; 38: 1055-1059.

23 Gold B, Merriam JE, Zernant J, Hancox LS, Taiber AJ, Gehrs K et al., AMD Genetics Clinical Study Group. Variation in factor B (BF) and complement component 2 (C2) genes is associated with age-related macular degeneration. Nat Genet 2006; 38: 458-462.

24 Despriet DD, Klaver CC, Witteman JC, Witteman JC, Bergen AA, Kardys I et al. Complement factor $\mathrm{H}$ polymorphism, complement activators, and risk of age-related macular degeneration. JAMA 2006; 296: 301-309.

25 Francis PJ, Schultz DW, Hamon S, Ott J, Weleber RG, Klein ML. Haplotypes in the complement factor $\mathrm{H}(\mathrm{CFH})$ gene: associations with drusen and advanced age-related macular degeneration. PLOS ONE 2007; 2: e1197.

26 Magnusson KP, Duan S, Sigurdsson H, Petursson H, Yang $\mathrm{Z}$, Zhao Y et al. CFH Y402H confers similar risk of soft drusen and both forms of advanced AMD. PLoS Med 2005; 3: e5.

27 Shuler Jr RK, Schmidt S, Gallins P, Hauser MA, Scott WK, Caldwell J et al. Phenotype analysis of patients with the risk variant LOC387715 (A69S) in age-related macular degeneration. Am J Ophthalmol 2008; 145: 303-307.

28 Shuler Jr RK, Schmidt S, Gallins P, Hauser MA, Scott WK, Caldwell $\mathrm{J}$ et al. Peripheral reticular pigmentary change is associated with complement factor $\mathrm{H}$ polymorphism $(\mathrm{Y} 402 \mathrm{H})$ in age-related macular degeneration. Ophthalmology 2008; 115: 520-524.

29 Tedeschi-Blok N, Buckley J, Varma R, Triche TJ, Hinton DR. Population-based study of early age-related macular degeneration: role of the complement factor $\mathrm{H} \mathrm{Y402H}$ polymorphism in bilateral but not unilateral disease. Ophthalmology 2007; 114: 99-103.

30 Dewan A, Bracken MB, Hoh J. Two genetic pathways for age-related macular degeneration. Curr Opin Genet Dev 2007; 17: 228-233.

31 Weger M, Renner W, Steinbrugger I, Köfer K, Wedrich A, Groselj-Strele A et al. Association of the HTRA1 -625G > A promoter gene polymorphism with exudative age-related macular degeneration in a Central European population. Mol Vis 2007; 13: 1274-1279.

32 Dasch B, Fuhs A, Schmidt J, Behrens T, Meister A, Wellmann $\mathrm{J}$ et al. Serum levels of macular carotenoids in relation to age-related maculopathy The Muenster Aging and Retina Study (MARS). Graefes Arch Clin Exp Ophthalmol 2005; 243: 1028-1035.

33 Dasch B, Fuhs A, Meister A, Behrens T, Krause K, Pauleikhoff $\mathrm{D}$ et al. [Association between classic cardiovascular risk factors and age-related maculopathy (ARM) Results of the baseline examination of the Munster Aging and Retina Study (MARS).]. Ophthalmologe 2005; 102: 1057-1063.

34 Dasch B, Fuhs A, Behrens T, Meister A, Wellmann J, Fobker $\mathrm{M}$ et al. Inflammatory markers in age-related maculopathy: cross-sectional analysis from the Muenster Aging and Retina Study. Arch Ophthalmol 2005; 123: 1501-1506.

35 Barrett JC, Fry B, Maller J, Daly MJ. Haploview: analysis and visualization of LD and haplotype maps. Bioinformatics 2005; 21: 263-265.

36 Leveziel N, Souied EH, Richard F, Barbu V, Zourdani A, Morineau G et al. PLEKHA1-LOC387715-HTRA1 polymorphisms and exudative age-related macular degeneration in the French population. Mol Vis 2007; 13: 2153-2159.

37 Purcell S, Cherny SS, Sham PC. Genetic Power Calculator: design of linkage and association genetic mapping studies of complex traits. Bioinformatics 2003; 19: 149-150.

38 Janssens AC, Aulchenko YS, Elefante S, Borsboom GJ, Steyerberg EW, van Duijn CM. Predictive testing for complex diseases using multiple genes: fact or fiction? Genet Med 2006; 8: 395-400.

39 Lin JM, Tsai YY, Wan L, Lin HJ, Tsai Y, Lee CC et al. Complement factor $\mathrm{H}$ variant increases the risk for early age-related macular degeneration. Retina 2008; 28 : 1416-1420.

40 Rothman KJ. Epidemiology: An Introduction. Oxford University press: New York, 2002.

41 Donoso LA, Kim D, Frost A, Callahan A, Hageman G. The role of inflammation in the pathogenesis of age-related macular degeneration. Surv Ophthalmol 2006; 51: 137-152.

42 Rodriguez DC, Esparza-Gordillo J, Goicoechea DJ, LopezTrascasa M, Sanchez-Corral P. The human complement factor $\mathrm{H}$ : functional roles, genetic variations and disease associations. Mol Immunol 2004; 41: 355-367.

43 Thakkinstian A, Han P, McEvoy M, Smith W, Hoh J, Magnusson $\mathrm{K}$ et al. Systematic review and meta-analysis of the association between complement factor $\mathrm{H} \mathrm{Y} 402 \mathrm{H}$ polymorphisms and age-related macular degeneration. Hum Mol Genet 2006; 15: 2784-2790. 